Cahiers du MONDE RUSSE

\section{Cahiers du monde russe}

Russie - Empire russe - Union soviétique et États indépendants

$55 / 1-2 \mid 2014$

Fiscalité, justice et conflit politique en Russie, premier tiers du XVIIle siècle

\title{
La politique ferroviaire russe et les financiers étrangers
}

Les ambiguïtés d'un projet réformateur après la guerre de Crimée Russia's railway policy and foreign financiers: A post-Crimean War ambiguous reform project

Juan Camilo Vergara

\section{OpenEdition}

Journals

Édition électronique

URL : http://journals.openedition.org/monderusse/7991

DOI : 10.4000/monderusse.7991

ISSN : $1777-5388$

Éditeur

Éditions de l'EHESS

Édition imprimée

Date de publication : 1 janvier 2014

Pagination : 149-180

ISBN : 9782713224409

ISSN : 1252-6576

Référence électronique

Juan Camilo Vergara, "La politique ferroviaire russe et les financiers étrangers », Cahiers du monde russe [En ligne], 55/1-2 | 2014, mis en ligne le 01 janvier 2017, Consulté le 01 mai 2019. URL : http:// journals.openedition.org/monderusse/7991; DOI : 10.4000/monderusse.7991

Ce document a été généré automatiquement le 1 mai 2019. 


\title{
La politique ferroviaire russe et les financiers étrangers
}

\author{
Les ambiguïtés d'un projet réformateur après la guerre de Crimée \\ Russia's railway policy and foreign financiers: A post-Crimean War ambiguous \\ reform project
}

Juan Camilo Vergara

1 La question des distances et des voies de communication dans un vaste pays comme la Russie du XIX ${ }^{e}$ siècle - un sixième de la planète - est au centre des préoccupations de l'État impérial, limité dans l'administration de son vaste territoire, alors même qu'il poursuit encore son expansion. Les besoins militaires, administratifs et économiques croissants rendent vitale l'existence de moyens de communication modernes et rapides.

2 Au lendemain de la désastreuse défaite dans la guerre de Crimée (1853-1856), l'état déplorable des voies de communication apparait comme la principale cause de la défaillance de l'armée russe. Le besoin d'effectuer des changements structurels se fait sentir dans plusieurs domaines. L'avènement d'Alexandre II, en 1855, marque l'ouverture d'une période de réformes dans laquelle les transports jouent un rôle fondamental. L'abolition du servage se profile à l'horizon, en même temps que le tsar effectue un rapprochement diplomatique avec la France, sous la responsabilité du ministre des Affaires étrangères, Aleksandr Gorčakov.

3 Dans l'espace des quelques mois qui suivent la capitulation russe, le monopole que l'État essayait de garder sur ses moyens de transport est ébranlé. L'effort de guerre a plongé la Russie dans une crise financière qui l'empêche de réformer ses moyens de transport sans le concours des puissances étrangères. En même temps, les capitaux européens entrent dans une phase favorable aux investissements à l'étranger et les marchés européens voient dans l'ouverture de la Russie de nouvelles opportunités.

4 Le nouveau tsar n'attend pas la signature du traité de paix de Paris, le 30 mars 1856 (selon le calendrier grégorien ${ }^{1}$ ), pour se tourner vers ses ennemis de la veille. Son objectif est de promouvoir une technologie de pointe pour renforcer l'administration de l'empire. Il profite du Congrès de Paris pour lancer un retentissant appel d'offres visant à la 
construction du premier réseau de chemins de fer russe. Mais la participation de puissants groupes financiers étrangers dans ce domaine stratégique peut constituer une menace pour l'autorité de l'état. Cet appel représente un avantage et un défi pour la souveraineté du tsar. En effet, l'interaction entre diplomatie et gestion économique, qui jaillit de l'arrivée des investisseurs étrangers, favorise l'apparition de puissants systèmes d'influence.

5 La construction du premier réseau russe commence en 1857 avec la fondation de la Grande société des chemins de fer russes (GSCFR). Cette compagnie française, avec un capital inédit de 275 millions de roubles, soit $72,5 \%$ de tous les capitaux par actions jamais investis jusque-là en Russie, s'engage dans la construction de $4280 \mathrm{~km}$ de chemins de fer ${ }^{2}$. Les ingénieurs projettent cinq lignes principales : Saint-Pétersbourg - Varsovie, avec son embranchement vers la Prusse, Moscou - Nižnij Novgorod, Libau - Kursk et Moscou - Théodosie. À cette date, la Russie comptait seulement $1003 \mathrm{~km}$ de chemins de fer et trois compagnies ferroviaires pour les lignes: Saint-Pétersbourg-Carskoe Selo, Varsovie - Vienne et Saint-Pétersbourg - Moscou. En l'espace de dix ans, la GSCFR devait réaliser quatre fois plus de voies que ce qui avait été construit en Russie depuis $1838^{3}$.

6 Très tôt, la compagnie doit faire face à l'animosité d'une partie du gouvernement et du corps des ingénieurs russes en constitution, qui cherche à reprendre sa direction et à évincer administrateurs et ingénieurs français. En même temps, à l'étranger, l'hostilité des banquiers Rothschild et Oppenheim entraîne un faible succès de cette compagnie dans les bourses européennes. La dévaluation du rouble et le manque de capitaux en 1860 poussent la GSCFR à la faillite. Elle renonce à la construction de la moitié de ses lignes et le réseau russe reste incomplet pendant la décennie suivante.

7 La GSCFR occupe donc une place particulière dans les réformes d'Alexandre II, d'autant plus qu'elle naît d'une concurrence internationale entre les milieux diplomatiques et financiers américains, prussiens, anglais et français. L'arrivée de groupes financiers de la taille des Rothschild, Pereire et Oppenheim dans le domaine ferroviaire place l'État en position d'arbitre et d'acteur. En effet, il assume ce rôle au moment où de puissants intérêts essaient d'influencer les prises de décisions de ministres et de fonctionnaires de la Direction générale des voies de communication (le GUPS) ${ }^{4}$. Les yeux de la Russie et de l'Europe se tournent entre 1856 et 1857 vers le combat auquel se livrent les maisons financières mondiales, avides des richesses et des matières premières russes. À la fois menaçantes et prometteuses, les réformes du nouveau monarque dans ce domaine industriel annoncent un basculement dans l'ordre militaire et économique européen. Comment contenir alors la Russie qui cherche à se doter d'une arme aussi puissante que les chemins de fer?

8 Les correspondances officielles et secrètes entre l'État russe et les maisons financières européennes et américaines sont des sources primordiales pour l'analyse. Elles permettent de déceler les ambitions qui dominent la question des chemins de fer dans cet empire ${ }^{5}$. Parmi les sources, les plus importantes - qui pourtant n'ont été que rarement exploitées par les historiens ${ }^{6}-$, se trouvent dans les archives de la compagnie à Saint-Pétersbourg?.

9 Notre propos est de comprendre les mécanismes qu'utilisent les maisons financières européennes pour influencer la politique d'Alexandre II dans ce domaine, et d'analyser en quoi ceci est révélateur d'une internationalisation du territoire russe. Pour identifier les intérêts en jeu nous devrons d'abord situer les frères Pereire dans le contexte russe. Nous pourrons ainsi observer pourquoi leur influence devient irrésistible en 1856. L'analyse des 
liens entre les Pereire et la Russie nous amènera ensuite à observer leur relation avec leurs concurrents et les ressources employées par chacun des rivaux pour faire pression sur le gouvernement russe. Les chemins de fer deviennent alors un objet de politique internationale, ce qui nous permettra d'évoquer dans un troisième moment le rôle du tsar et de ses ministres. L'État essaie de canaliser cet élan international en sa faveur, en vue de rendre plus réelle et performante sa présence dans les régions de l'empire.

\section{Les frères Pereire, l'arrivée d'un groupe financier en Russie}

10 Isaac et Émile Pereire sont, dans l'histoire industrielle russe, les fondateurs d'une compagnie ferroviaire sans précédent. Ils réussissent à fédérer autour d'eux un groupe de banquiers de taille internationale, parmi lesquels se trouvent Louis Fould, Henri Hottinguer et Auguste Thurneyssen à Paris, Thomas Baring à Londres, Mendelsshon et Cie à Berlin, Hope et Cie à Amsterdam et Aleksandr Stieglitz à Saint-Pétersbourg. Le capital qu'ils réunissent, le contexte dans lequel leur compagnie apparaît et les controverses qu'elle suscite font de la GSCFR une société unique jusqu'alors en Russie. Les Pereire mettent fin à la prépondérance de l'État qui pesait depuis vingt ans sur le domaine ferroviaire, et s'imposent face à une concurrence féroce à laquelle participent les grandes maisons financières d'Europe et des États-Unis ${ }^{8}$.

Leur influence en Russie commence bien avant l'idée même de fonder la GSCFR, quand leurs entreprises en France marquent une génération de réformateurs en Russie. Aux yeux de certains Russes, dont l'influent statisticien Ludvig Tegoborskij', la fondation du Crédit mobilier et la construction du chemin de fer de Paris - Saint-Germain apparaissent comme un exemple à suivre. Les deux frères font partie d'un groupe d'investisseurs critiques de ce qu'ils appellent la finance conservatrice, les « oisifs ${ }^{10}$, réticents à mettre en péril leurs activités foncières. Dans le développement de leurs activités dans le secteur bancaire, que l'exemple du Crédit mobilier illustre bien ${ }^{11}$, ils critiquent sans cesse les « rentiers parasitaires » qui ne donnent pas de contrepartie à l'économie ni une véritable production. Ils cherchent alors à réorienter l'épargne vers la croissance, d'où leurs activités de plus en plus audacieuses qui les amènent jusqu'en Russie. Dès leurs premiers pas auprès des Rothschild en $1832^{12}$ jusqu'à leurs activités en Espagne et en Italie, ils font preuve d'un esprit d'initiative qui rivalise en 1856 avec celui des Rothschild ${ }^{13}$.

12 Si la carrière d'Émile et Isaac Pereire est inséparable de la construction des chemins de fer français, elle l'est aussi de l'industrie ferroviaire russe. La fondation du Crédit mobilier, le 18 novembre 1850, et leurs activités dans d'autres pays européens attirent l'attention des défenseurs des chemins de fer en Russie. Leurs idées apparaissent en Russie comme un possible remède contre le retard industriel du pays. Le prestige de cette banque et de ses fondateurs se fait sentir d'amples façons en Russie, comme le laisse comprendre l'Essai sur le Crédit mobilier de Tegoborskij ${ }^{14}$. Des sources françaises font écho aux opinions russes sur la banque des Pereire. En effet, le nom de L. Tegoborskij est cité dans certains imprimés de l'époque tels que le Grand dictionnaire universel Larousse du xixe siècle, qui mentionne ses travaux sur le Crédit mobilier ${ }^{15}$.

13 Le Crédit mobilier est le point d'entrée en Russie de l'industrie ferroviaire française et se trouve au cœur de l'internationalisation de la question des chemins de fer russes ${ }^{16}$. Il est bien l'un des facteurs qui amènent à la fondation de la GSCFR. Cependant, plusieurs forces 
limitent son influence en Russie à partir de 1856, notamment parce que la stratégie de l'État russe est d'ouvrir la construction de chemins de fer privés à la concurrence internationale. Parmi ces forces se trouve une partie des actionnaires de la banque, réticents aux investissements en Russie. Une crise financière s'annonce en Europe, alors que la Russie et « les chemins de fer russes étaient aussi l'objet de longs commentaires [de la part des actionnaires de la banque ( $\mathrm{NdA})]$; mais la part du Crédit mobilier dans les opérations relatives à ces affaires était passée sous silence $\aleph^{17}$. Un rapport de l'ambassade française à Saint-Pétersbourg sur les négociations des frères Pereire en Russie fait référence à Émile Pereire, «directeur de la Compagnie du Crédit mobilier, dont la personnalité paraît aujourd'hui assez effrayer ceux qui se montraient naguère les plus disposés à entrer dans les chemins de fer russes ${ }^{18}$.

Bien que la concession d'une grande compagnie de chemins de fer en Russie semble une bonne affaire pour les capitaux européens de l'époque, les possibilités de succès font l'objet de doutes. Si, pour certains, le territoire russe apparaît comme un «Far East » européen, en l'attente d'hommes courageux qui puissent mettre en valeur son potentiel, des réticences s'expriment, même aux endroits les plus inattendus. Ainsi, une lettre du banquier russe Aleksandr Stieglitz ${ }^{19}$ nous apprend que le Crédit mobilier, la banque sur laquelle les Pereire appuient leurs propositions, «est en opposition directe avec ces Messieurs de Paris, quoiqu'elle leur doive pour ainsi dire sa création $»^{20}$.

Alors qu'il représente un obstacle pour la conclusion d'un accord avec l'État russe, le Crédit mobilier ne cesse d'attirer l'attention en Russie. Les articles des frères Pereire dans des journaux saint-simoniens comme Le Globe trouvent en Russie des lecteurs attentifs. Certains journaux russes deviennent spontanément les porte-parole des visions financières des Pereire. Les principaux articles en faveur de leurs activités en Russie paraissent dans des journaux contrôlés par de grands entrepreneurs russes ${ }^{21}$. L'idée d'introduire des banques mutuelles séduit ces derniers dans la mesure où

le mérite des sociétés mobilières de crédit consiste en ce qu'elles brisèrent le jeu et le monopole courtier des pouvoirs financiers. [...] Quels bénéfices ont-ils gagné grâce à l'accomplissement de simples devoirs caissiers, grâce à leur signature dans des projets qu'ils n'ont même pas rédigés et pour lesquels ils n'ont pas pris de responsabilités juridiques ou factuelles. Dans la période où les Rothschild et d'autres monopolistes semblables régnèrent, plus que jamais, dominait un système de charges féodales qui ne correspondait à aucun effort de contrepartie. La lune de miel du royaume de l'argent s'est achevée et qui est le responsable ? C'est la faute aux Crédits mobiliers qui ont brisé le pouvoir patriarcal des fils d'Abraham..$^{22}$

La ferveur entrepreneuriale des Pereire coïncide avec une concurrence financière de plus en plus présente au niveau international. Ce ne sont plus seulement des individus qui entrent en concurrence au sein du marché ferroviaire européen, mais des gouvernements qui constituent des groupes de pression en alliance avec des groupes financiers. Ces liens sont d'autant plus évidents que, dans le cas de la Russie, les frontières entre le diplomatique et le financier s'effacent; ils trouvent un objectif commun : le territoire et les ressources russes. Ainsi, nous pouvons parler dans notre domaine d'une coexistence d'intérêts privés et officiels chez les acteurs en question.

17 Le cas du comte de Morny ${ }^{23}$, lui-même actionnaire du Crédit mobilier, nous montre le type de concurrence qui se met en place en 1856. En effet, ses contemporains affirment à l'époque qu' i il suffit que l'on entende prononcer d'un air entendu, le fameux "Morny est dans l'affaire" pour que ladite affaire attire capitaux et obtienne les autorisations 
nécessaires $»^{24}$. Sa relation avec les Pereire est un exemple parlant des frontières poreuses entre les affaires d'État et les affaires privées au XIX ${ }^{e}$ siècle.

Les Pereire arrivent en Russie en septembre, afin de donner une impulsion décisive à leurs projets russes. Ils font preuve de détachement envers le grave retard des transports en Russie et soutiennent le 15 septembre que «la résolution de ce départ n'a été que l'expression d'une juste déférence envers le Gouvernement de S. M. l'Empereur $»^{25}$. Mais, en réalité, ils sont bien plus impliqués qu'ils ne le pensent en raison de l'appui que leur prête le gouvernement français.

19 Le comte de Morny devient l'ambassadeur français auprès du tsar peu après que la Russie a lancé son appel d'offres pour la construction du réseau ferroviaire. Il est présent à Saint-Pétersbourg pendant les négociations. Son comportement est ambigu, dans la mesure où ses actions dans le Crédit mobilier sont aussi en jeu. Ses affaires relient entre eux le milieu diplomatique, le monde entrepreneurial et la famille impériale française. Il est le demi-frère de Napoléon III et sa présence en Russie démontre la confiance dont l'empereur fait preuve à son égard. Le rôle de la France se partage alors entre la politique de l'État français et le profit économique personnel.

$20 \mathrm{Au}$ début de la guerre de Crimée, Morny ne fait pas seulement partie du cercle des Pereire, mais nous le retrouvons dans d'autres affaires comme celle de la spéculation de la concession de chemin de fer du Grand Central, dans laquelle le Crédit mobilier fut aussi impliqué26. "[...] L'entrée de M. le comte de Morny dans le conseil d'administration du Crédit mobilier devait sans doute se rattacher à cette opération dont les résultats furent considérables ", ce qui ne passa pas inaperçu à l'époque ${ }^{27}$.

21 Pour le gouvernement russe, il devient impératif de canaliser les forces en place et de s'interroger sur l'influence du gouvernement français dans cette affaire. Le GUPS produit un rapport le 17 octobre $1856^{28}$ qui pose les principales questions sur les chemins de fer :

Les chemins de fer sont-ils nécessaires et urgents pour la Russie ? Pouvons-nous achever rapidement leur construction sans le concours des étrangers? Pouvons-nous les terminer rapidement et en de bonnes conditions? Notre performance sera-t-elle satisfaisante si on la compare aux propositions des banquiers Pereire? Pourrait-on admettre des chemins parallèles [à ceux construits par les Pereire, (NdA)] ? Peut-on appliquer les mêmes tarifs que dans le chemin de fer de Moscou ? Peut-on conclure le marché avec les Pereire ?29

Ce rapport montre comment l'État essaie de chercher des mécanismes pour garder un contrôle sur la future GSCFR et maintenir une marge de manœuvre en cas de besoin. Ceci se voit principalement dans son questionnement sur la construction d'éventuelles lignes ferroviaires parallèles, qui permettraient au gouvernement de réduire sa dépendance vis-à-vis des administrateurs français. En effet, si le rôle ambigu du comte de Morny est à nos yeux important à signaler, pour l'État russe ce n'est pas un secret que le groupe des Pereire est placé sous l'égide du gouvernement de Napoléon III.

Les réticences de l'État face aux pouvoirs qu'aurait une telle compagnie deviennent évidentes si on les compare aux aspirations des frères Pereire. Ces derniers voient dans la GSCFR, non pas une affaire isolée, mais une porte d'entrée en Russie. En même temps que le territoire russe est un terrain de dispute politique et économique entre les puissances, l'objectif des investisseurs est l'ouverture d'un nouveau marché qui permette de s'introduire dans d'autres secteurs du pays. Les Français observent les transports russes comme le pilier d'un édifice en construction. Ils proposent un réseau centralisé sous leur direction, la privatisation de la ligne Nikolaevskaja (Saint-Pétersbourg - Moscou) et, de 
préférence, « l'obtention de la concession de houillères et d'usines dans le but de faciliter l'extension à donner en Russie au système des voies de communication $»^{30}$.

Durant les négociations, l'État russe cherche à donner des contours clairs à la GSCFR et à contenir ainsi son indépendance. Pour leur part, les Pereire utilisent diverses stratégies pour étendre leur influence à d'autres secteurs de l'économie russe. Le principal succès des Français est la constitution d'un quasi-monopole dans le domaine des transports. Leur impétuosité les pousse, en mars 1856, à proposer de fonder un Crédit mobilier russe, comme cela avait été le cas en Espagne. À ce propos, A. Stieglitz explique dans une lettre adressée au gouvernement que «leur pensée serait d'établir tout d'abord une grande institution de crédit que l'on pourrait appeler: Société Générale industrielle et financière $»^{31}$. Il soutient qu'il serait bénéfique pour l'industrie d'introduire une "société de crédit industriel sous l'expression la plus perfectionnée de cette association des capitaux $\aleph^{32}$. À terme, la banque devrait commencer à fonder spontanément des entreprises d'utilité publique, émettre des obligations et s'associer à la construction ferroviaire sans la subvention de l'État. La proposition est claire, l'État n'a qu'à attendre que des investissements massifs révolutionnent l'économie russe.

Le refus ne se fait pas attendre de la part d'un État russe qui n'a ni l'intérêt ni l'habitude de rester en position d'observateur. Le gouvernement veut garder la mainmise sur les limites juridiques des compagnies ferroviaires en négociation, mais il reste plus discret dans d'autres domaines. Par exemple, en 1856, il est largement absent des choix techniques sur les lignes à construire. Les ingénieurs français, anglais, allemands et américains proposent des modifications aux lignes projetées auparavant en Russie. L'État est pris entre ses besoins et ses devoirs, ce qui explique qu'il ne devient qu'un acteur de plus menant ses propres explorations de terrain. Dans le cas des Pereire, des ingénieurs des Ponts et Chaussées dirigent les études qui aboutissent à une proposition formelle le 27 juin 1856. A. Stieglitz se charge de présenter au gouvernement les plans de construction des lignes Saint-Pétersbourg - Varsovie (avec son embranchement à la frontière prussienne), Moscou - Théodosie (avec son embranchement au fleuve Dniepr), Moscou - Nižnij Novgorod et Kursk - Libau ${ }^{33}$. Ces plans modifient des lignes largement discutées en Russie depuis les années $1820^{34}$, y compris par Aleksandr Puškin ${ }^{35}$. D'ailleurs, ce sont des ingénieurs français réputés qui donnent naissance au débat durant les années $1820^{36}$, avec le Journal des voies de communication, revue bilingue russo-française éditée à Saint-Pétersbourg. Ils forment la première génération d'ingénieurs russes.

Dans les hautes sphères de l'État, le dilemme est de choisir entre le risque de faillite d'une compagnie privée d'une telle ampleur (avec un capital de 275 millions de roubles), et l'urgence de moderniser les transports. La correspondance entre le baron Zeebah ${ }^{37}$ et le prince Aleksej $\mathrm{Orlov}^{38}$ en est un exemple ; le rapprochement avec la France semble se faire naturellement, mais les méfiances face à l'ennemi de la veille se font sentir. Faisant

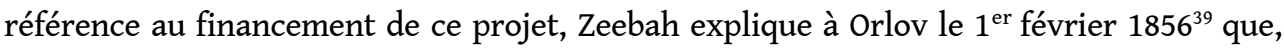
"sous l'un et l'autre rapport, la France m'a toujours semblé le pays où Vous deviez chercher Votre point d'appui $»^{40}$. Le gouvernement russe s'attend d'ailleurs à ce que les Pereire soient les premiers à faire des propositions dans les mois à venir. Ils sont présentés comme « les seuls hommes capables de mener une entreprise aussi colossale à bonne fin [...] ce sont des gens calmes, modestes, nullement autoritaires $»^{41}$. Les mots de Zeebah rendent évidente la situation privilégiée qu'occupent les Pereire dans le paysage ferroviaire russe. 

et d'autres gouvernements s'allient pour leur barrer la route, comme c'est le cas des Américains avec Tal Shaffner, des Anglais avec les Rothschild et des Allemands avec les Oppenheim. La constitution de ces lobbies mobilise la société russe qui se méfie du contrôle européen sur le réseau russe. Le GUPS, pourtant l'organisme chargé de mener à bien la construction des chemins de fer, se présente comme le défenseur de la souveraineté russe. Il assume un rôle de contrepoids dans les négociations, ce qui annonce une rivalité future avec la GSCFR.

Les contacts pour la constitution d'une compagnie ferroviaire commencent avant la fin du Congrès de Paris, ce qui donne aux parties le temps de faire les calculs et les études nécessaires pour les discussions à venir. Néanmoins, dès cette époque, et sans qu'aucun des investisseurs ne le note, Konstantin Čevkin ${ }^{42}$ exprime ses réserves. Comme il le fera par la suite, il cache son hostilité sous la couverture d'un langage officiel. Le 5 février 1856 , il admet que le gouvernement appuie la construction du réseau et que

les propositions que Vous [Stieglitz, NdA] voulez bien m'indiquer sont dignes de toute considération, et une fois que les circonstances politiques le permettront, nous leur accorderons assurément l'accueil et l'examen réfléchi qu'elles méritent, car notre désir prononcé est, comme Vous le savez, d'accepter franchement le concours des Compagnies particulières, qui voudraient se charger de l'établissement et de l'exploitation de nos principales lignes de chemin de fer. ${ }^{43}$

Bien que ces mots semblent indiquer qu'il attend d'éventuelles propositions des Pereire, il fait exprès de ne pas parler de compagnies particulières et étrangères. Cet oubli nous permet de comprendre qu'en Russie, simultanément, commence à se constituer un groupe d'investisseurs russes fortement opposés au contrôle des transports par des compagnies étrangères. Avant même que les Pereire et leurs concurrents ne réussissent à présenter des demandes formelles de concession devant le gouvernement russe, la politisation de la question ferroviaire s'approfondit. Ceci s'explique par l'introduction en Russie d'un système de concurrence entre Européens dont nous devons identifier les acteurs.

\section{La concurrence internationale}

A. Stieglitz arrive au printemps 1856 en France pour attirer l'attention de plusieurs grands investisseurs du monde ferroviaire européen. Le Congrès de Paris semble l'occasion idéale pour faire la promotion du potentiel ferroviaire de l'empire et des bénéfices que pourrait tirer l'Europe des matières premières russes. La Russie d'Alexandre II se veut avant tout réformatrice et essaie d'inspirer confiance aux investisseurs après la guerre de Crimée. Dans le domaine ferroviaire, les attributs qu'elle veut exhiber en Europe s'atténuent avec l'impossibilité financière et technique de moderniser les transports.

31 L'ouverture des négociations attire l'attention de nombreux investisseurs mondiaux, conscients des énormes revenus d'une telle affaire. Mais les défis que pose la construction du réseau poussent les groupes financiers d'alors à former des alliances. On en compte principalement quatre. La première d'entre elles se forme en 1855 autour de Tal Shaffner, constructeur de télégraphes durant la guerre de Crimée et représentant en Russie de la Compagnie américaine de télégraphes ${ }^{44}$. Le gouvernement prussien soutient la deuxième alliance qui réunit, autour de la maison Oppenheim, plusieurs investisseurs allemands et 
belges, ainsi que le russe Aleksandr A. von Ljarljarskij ${ }^{45}$. La troisième se forme avec l'appui du groupe financier des Rothschild et de l'anglais Whitehall. La dernière se crée à partir des proches du Crédit mobilier comme les Pereire, Hottinguer, Fould et Thurneyssen entre autres.

Face à l'intérêt qu'éveille A. Stieglitz en 1856, le gouvernement russe ne peut s'empêcher de constater à quel point la modernisation des transports dépend des négociations avec ces groupes financiers. Durant la décennie suivante, E. Lamanskij, le futur directeur de la banque d'État, le fait savoir à ses contemporains. Il indique que «nulle part ailleurs, même à l'étranger, nous ne voyons d'autres formes d'entreprises privées qui puissent réunir des capitaux de la manière dont le font les entreprises d'actionnaires ${ }^{46}$. En même temps, le monde industriel européen de l'époque ne peut pas ignorer la dimension de ce qu'A. Stieglitz propose de construire. La fondation de la GSCFR et l'ouverture du marché ferroviaire russe ne constituent pas seulement une opportunité d'investissements sans limites. Elles représentent l'un des investissements à l'étranger les plus importants à réaliser par l'industrie européenne, puisque

les chemins de fer russes n'avaient pas réalisé entièrement les espérances qu'on aurait pu concevoir au début des négociations; des sommes considérables, telles qu'aucune compagnie n'en avait pu réunir jusqu'alors, avaient été ou auraient été nécessaires, pour la construction de cet immense réseau qui devait mettre en communication la mer Noire et la Baltique [...]. La Compagnie fut épouvantée des obstacles qui surgissaient de toutes parts, ces obstacles venaient surtout de la difficulté de se procurer le capital nécessaire : c'est en vain qu'on avait fait appel à diverses reprises à tous les capitaux de l'Europe. 500 millions avaient été déjà dépensés sur le chemin de fer de Moscou à Nižnij-Novgorod et sur la section de Saint-Pétersbourg à Varsovie, avec prolongement jusqu'à la frontière prussienne. Des difficultés de tout genre avaient surgi entre les administrateurs de la Compagnie et le gouvernement impérial de Russie [...]. ${ }^{47}$

\section{Les propositions des Américains}

L'ampleur des travaux que la Russie envisage de réaliser en 1855 pousse les Américains à élaborer, les premiers, des propositions. Alors que la guerre empêche la Russie de négocier avec l'Europe la fondation d'une compagnie ferroviaire, Tal Shaffner suggère au gouvernement russe de se tourner vers les États-Unis. Il s'agit de la première proposition d'envergure, promettant la construction des lignes imaginées par les Russes depuis les années 1830. Shaffner arrive en Russie en août 1854 pour travailler sous la direction du comte P. Kleinmichel, directeur du GUPS sous Nicolas $\mathrm{I}^{\mathrm{er}}$. Sa mission est alors de construire un réseau télégraphique qui puisse pallier les déficiences des communications apparues durant la guerre de Crimée. Durant ses travaux, il décide de proposer au gouvernement de fonder une compagnie ferroviaire d'envergure. En août 1855, il entre en contact avec le comte A. Orlov pour faire passer son message à l'empereur. Il affirme dans ses correspondances qu'il a l'appui du président des États-Unis, avec lequel il s'est entretenu personnellement. En effet, Shaffner affirme que le président s'est dit prêt à soutenir ce projet en Russie et à briser le blocus maritime dans la mer Baltique. De retour à Washington, Shaffner écrit :

J'ai à nouveau soumis mes plans à la considération du président des États-Unis et je ne dois pas me tromper si je dis que le gouvernement me défendrait dans la rupture du blocus dans la Baltique : j'étais satisfait de trouver dans le cabinet américain une 
grande sollicitude pour la conclusion de mes plans - pour des considérations sociales et politiques. ${ }^{48}$ Face aux promesses de Shaffner, le gouvernement lui demande de fournir des garanties explicites pour donner suite à ses propositions. À l'époque, il n'a probablement pas pris la mesure de ce qui commence à changer en Russie avec la défaite en Crimée, quand le nouveau règne se tourne vers l'Europe (et particulièrement vers la France) pour rattraper son retard technologique. Dans cette mesure, l'ingénieur américain exprime dans ses lettres les regrets que lui inspire la lenteur de la contrepartie russe. Il critique ouvertement la prudence du gouvernement vis-à-vis des investisseurs américains et le fait que le Comité de ministres ignore l'ambassadeur américain à Saint-Pétersbourg. Répondant aux doutes exprimés par Čevkin sur le sérieux de ses associés, T. Shaffner s'emporte :

Je ne comprends pas la raison de poser une telle question. Ce n'est pas conforme aux usages du business en Amérique. Mes banquiers font partie de ma Compagnie. Je suppose que la bonne question aurait dû être de s'informer de la solidité et de la compétence de mes associés, dont je refuse de donner les noms pour ces mêmes raisons.

Je considère le ministre américain à Saint-Pétersbourg comme une personne bien plus importante à consulter sur la solvabilité et l'intégrité de la compagnie, que le ministre russe dernièrement nommé à Washington ; mais, en même temps, je serais 
ravi de me conformer au rapport du ministre russe s'il était possible de l'obtenir dans les plus brefs délais.

Je considère comme suffisante l'opinion des représentants officiels de mon pays ; et si le gouvernement ne peut pas compter sur leur parole, comment devrais-je obtenir sa confiance? Dans ce cas, comment pourrais-je signer un contrat avec la Russie?

Je vous présente une liste des pertes et dépenses que j'ai subies depuis mon arrivée. Perte d'un an de salaire en tant que lieutenant-général des Télégraphes électriques $12000 ; 8$ mois de salaire d'ingénieur 4000 ; dépenses de voyage 7600 ; total : 23600 dollars. ${ }^{54}$

Shaffner emploie un ton qui dépasse de loin celui employé par les investisseurs français au moment où Čevkin met en péril la conclusion d'un contrat. Nous comprenons alors que le tsar veut en effet attendre la fin du conflit pour faire plier Shaffner et lui faire baisser ses propositions. Cet exemple montre comment le gouvernement russe cherche, après 1855 , non pas le concours de grands ingénieurs ou l'expérience d'entreprises ferroviaires d'Europe ou des États-Unis ${ }^{55}$, mais les ressources financières pour garantir la modernisation de son système de transports.

Le gouvernement russe reste dans l'attente d'une proposition plus avantageuse comme celle qu'il reçoit l'année suivante de la part des investisseurs européens. Le GUPS en est conscient et finit par donner à Shaffner une réponse officielle négative après plusieurs mois d'attente :

À l'époque actuelle il ne serait guère possible de passer un contrat pour cette opération, vu que ni les recherches locales, ni les projets et devis ne sont achevés [...

]. Le Gouvernement ne prend aucun engagement, tant que ces capitalistes ne présenteront pas au Gouvernement leurs propositions. ${ }^{56}$

En effet, depuis le 23 août 1855, le Comité pour les chemins de fer arrive à la conclusion que le blocus maritime rend impossible la prise en considération des propositions de Shaffner ${ }^{57}$.

Deux ans avant le lancement de l'appel d'offres pour la construction de son réseau ferré, les Américains trouvent une Russie toujours réticente à établir des négociations, mais la situation financière ne cesse d'empirer entre 1855 et 1856. Ce n'est qu'en raison du changement de règne et de la défaite en Crimée que le besoin de rouvrir les négociations devient primordial en 1856.

\section{Les propositions belges et allemandes}

41 Le deuxième groupe qui s'offre à construire le réseau ferré russe se forme autour de la famille Oppenheim à Cologne. Ils entrent en alliance avec des entrepreneurs belges afin de constituer un groupe capable de séduire financièrement le gouvernement russe. Tout comme l'a fait Shaffner deux ans auparavant, ils élaborent une carte ferroviaire qui correspond largement aux cartes imaginées au cours des vingt années précédentes. Derrière eux se tiennent les gouvernements de Prusse et de Belgique, dont Florentin-Xavier Brower de Hogendorp ${ }^{58}$ devient le correspondant principal. Les Oppenheim s'allient avec une diversité d'investisseurs de taille qui rivalisent avec les Pereire. Parmi eux se trouvent les principaux industriels et banquiers de Belgique et des pays allemands comme la banque Schaaffhausen ou J.R. Bischoffsheim en Belgique. Ces derniers sont profondément liés à des investisseurs français et prussiens ${ }^{59}$. Leurs troupes se renforcent avec l'arrivée de la Banque de commerce et d'industrie de Darmstadt, celle 
de Philippe Schmidt avec une association de banquiers à Francfort, celle de la Société générale de commerce de Berlin et de la banque du Midi de l'Allemagne parmi d'autres ${ }^{60}$.

Leurs propositions arrivent en Russie autour du 27 septembre 1856, c'est-à-dire plus de six mois après que les Pereire ont manifesté leur désir de construire le réseau. De manière générale, ils proposent de construire les mêmes lignes que Shaffner, et non pas que les Pereire ${ }^{61}$. Mais ils rejoignent les Pereire dans leur intention d'acheter à l'État la ligne entre Moscou et Saint-Pétersbourg et l'usine galvanoplastique de Saint-Pétersbourg ${ }^{62}$. Cette affaire leur permettrait de créer un monopole qui garantirait le succès de la compagnie. Si Shaffner avait proposé de construire les lignes en cinq ans, le groupe Oppenheim doit faire des propositions plus réalistes puisqu'il est au centre d'une concurrence féroce entre maisons financières européennes. C'est alors qu'ils envisagent de terminer le réseau dans un délai de dix ans.

Ils soutiennent leurs propositions avec un capital de 268 millions de roubles, deux millions de plus que les Américains, "par l'émission d'actions au porteur ou obligations (en diverses séries) de 125 roubles l'action. L'amortissement ne commencera pas avant 45 ans [au lieu de 20 ans pour Shaffner $(\mathrm{NdA})$ ] et ne durera pas au-delà de 90 ans $»^{63}$. Les Américains proposaient une concession de 40 ans et un possible amortissement après 20 ans. Cette différence nous laisse comprendre qu'avec la fin de la guerre, les maisons financières ont l'intention de rester longtemps en Russie ${ }^{64}$. Il n'y rien d'extraordinaire à voir dans les propositions des Européens une durée de concession de 90 ans. Les Européens essaient de mettre en place des systèmes financiers qu'ils ont déjà réalisés dans leurs pays, ainsi que de profiter de l'ouverture économique de la Russie. En effet, Brower de Hogendorp fait comprendre à Čevkin que l'un des objectifs des Belges dans la construction du réseau russe est de permettre l'entrée de produits en provenance de Belgique. Il est chargé « d'une mission qui a pour objet de rechercher quels sont les produits de l'industrie belge dont l'importation pourrait plus particulièrement se faire avec avantage dans l'Empire russe $»^{65}$. Dans ce domaine, l'étude de marché que réalise l'ingénieur belge Adolphe Le Hardy de Beaulieu ${ }^{66}$, pour le compte d'investisseurs belges, décrit les chemins de fer russes comme la porte d'entrée au marché russe.

De leur côté, les banquiers allemands commencent, dès août 1856, à augmenter leur pression sur le GUPS. La correspondance entre Jan von Wendelstadt, directeur de la banque de Darmstadt, et Čevkin nous en donne un exemple. Le 25 septembre 1856, Wendelstadt écrit au directeur des voies de communication : « Nous espérions alors, voir promptement réalisé notre désir de construire en Russie un chemin de fer modèle pour nous montrer dignes de la confiance dont le Gouvernement de Sa Majesté aurait voulu nous honorer ${ }^{67}$. En même temps, et comme nous le verrons pour d'autres rivaux des Pereire, le groupe Oppenheim fait tout pour empêcher les Français et le comte de Morny de s'imposer dans les négociations.

Pour soutenir leurs intérêts et contrecarrer l'influence des Français, le gouvernement prussien et les investisseurs de Belgique et d'Allemagne décident d'envoyer des représentants à Saint-Pétersbourg. Ils arrivent simultanément avec les Pereire, en septembre 1856. Le 6 septembre 1856, Wendelstadt écrit à nouveau au GUPS. Il l'informe qu'il a reçu les pleins pouvoirs de la part de ses associés pour négocier directement avec le gouvernement russe la formation d'une compagnie germano-belge. Ces clients se sont " occupés de la formation d'une société assez forte pour faire une pareille entreprise " Le 12 septembre de la même année, Čevkin se rend compte que la partie prussienne est prête à faire des explorations de terrain et à donner un devis estimatif pour la ligne de 
Varsovie à Saint-Pétersbourg, celle qui naturellement les intéresse le plus en raison de son embranchement vers la Prusse ${ }^{69}$. Mais ce n'est qu'avec l'arrivée de Simon Oppenheim le 17 septembre 1856 que le groupe donne une véritable impulsion à ses propositions. S. Oppenheim ne perd pas de temps et envoie un télégramme le jour même de son arrivée à Čevkin: "arrivé à l'instant avec dépêches de mon gouvernement, je prie Votre Excellence de donner ordre pour me permettre de partir immédiatement par train spécial pour Moscou $»^{70}$.

Mais le gouvernement russe essaie de recevoir de la part des Oppenheim des propositions plus intéressantes que celles des Pereire. D'une part, son objectif est de pousser les Français à réduire la jouissance de la concession à 85 ans. D'autre part, le gouvernement impose au groupe allemand un délai de 10 ans pour terminer le réseau, sous peine de voir l'État annuler le contrat unilatéralement ${ }^{71}$. Le groupe germano-belge est conscient que, faute de réussir à se démarquer des Pereire, la partie serait perdue.

Les questions politiques commencent alors à jouer un rôle central dans les négociations. Nous apprenons que le 10 octobre 1856 le groupe Oppenheim se sait déjà vaincu face aux Pereire et élabore tout un argumentaire à leur encontre. Il pose un dernier recours pour, au moins, participer au financement des chemins de fer russes aux côtés des Pereire. Leur idée, « c'est un arrangement d'après lequel la Compagnie Allemande-Belge serait admise à prendre, dans une certaine proportion, part à l'entreprise [des Pereire $(\mathrm{NdA})] \aleph^{72}$, en référence à la GSCFR. Leurs critiques nous donnent une idée des rivalités entre les puissances européennes pour la domination du marché ferroviaire russe et, plus généralement, pour la pénétration sur le territoire de l'empire. Pour le groupe des Oppenheim, l'appel d'offres est « la plus grande affaire industrielle qui ait jamais été faite. En tout temps, ce ne serait pas trop de la réunion de toutes les forces financières pour la mener à bonne fin $~^{73}$. Dans leur intérêt à convaincre le GUPS de la nécessité de faire participer ce groupe aux investissements des Pereire, le groupe germano-belge nous révèle la place qu'occupe cette affaire dans les mentalités de l'époque. Pour soutenir leurs propositions, ils accusent les Pereire d'entraîner tout le monde dans une «fièvre de spéculation, qui agite aujourd'hui, une partie de l'Europe $»^{74}$, qui met en risque les capitaux européens et les chemins de fer russes. Ils se portent garants de la prudence, puisque

où sévit-elle [la spéculation $(\mathrm{NdA})$ ] avec le plus de violence et le plus longtemps ? Ce sera assurément là où la spéculation a été la plus folle, là où se sont formées le plus d'entreprises éphémères. Or, c'est assurément en France que la spéculation a été la plus effrénée. Dans cet état de choses, n'est-il pas désirable que l'affaire soit soutenue par des forces financières des pays qui sont dans une situation plus saine?

La GSCFR apparaît dominée par l'influence de la France et des Pereire, et c'est à ce pays que l'on s'attaque puisque, comme nous l'avons observé, on n'assiste plus à la concurrence de maisons financières, mais à celle des puissances mondiales. Malgré le désir évident d'investir en Russie, les rivaux des Pereire commencent, dès janvier 1857, une campagne de diffamation dans laquelle on met en garde les marchés européens contre les chemins de fer russes. Les critiques à l'égard des Pereire ne se limitent pas au domaine de l'économie ou de l'industrie. Elles passent constamment par la politique, puisqu'en

Allemagne et en Belgique la crise financière sévit avec beaucoup moins d'intensité qu'en France [...] l'affaire est colossale ; il s'agit de plus d'un milliard de francs ; son 
opération doit s'étendre sur dix années. Quels seront les événements politiques pendant cette période ? $^{76}$

\section{gouvernement, qui le presse de conclure un accord avec les Français. Face aux} suggestions du groupe germano-belge, il donne une réponse définitive le 16 octobre 1856. Il annonce « qu'il n'est point dans les habitudes du Gouvernement russe d'intervenir dans de semblables transactions $»^{77}$ en même temps qu'il exprime, ce même jour qu'il "n'y a plus lieu de donner suite à vos propositions $»^{78}$.

51 La Prusse et la Belgique sont deux des principaux acteurs dans la réforme des transports russes. Cependant, le renom du Crédit mobilier, des frères Pereire et l'ancien lien dans le domaine de l'ingénierie entre la France et la Russie diminuent la marge de manœuvre du groupe Oppenheim. Dans cette rivalité internationale, l'Angleterre joue aussi un rôle politique majeur.

\section{Les Rothschild sous la protection anglaise}

Les liens entre les Rothschild et les chemins de fer russes sont bien plus anciens que ce que les spécialistes affirment. Ils n'ont pas attendu la période des réformes d'Alexandre II pour se réaliser. En effet, dès le début de la construction de la ligne Nikolaevskaja, E. Kankrin ${ }^{79}$ fait le tour de l'Europe à la recherche de financements. Il entre alors en relation avec les principales maisons financières du continent. Durant son passage à Londres, en janvier 1842, le ministre rencontre les Rothschild en vue d'un éventuel financement de la ligne Saint Pétersburg - Varsovie ${ }^{80}$. En même temps, il fait une proposition similaire à la maison Hope d'Amsterdam ${ }^{81}$. Pour appuyer ses demandes, E. Kankrin se rapproche d'A. Stieglitz qui facilite le grand emprunt russe pour l'achèvement du chemin de fer entre les deux capitales. Peu de mois après le passage de Kankrin, « le Baron A.L. Stieglitz avait été envoyé à Londres et un agent de la maison Rothschild était à Saint-Pétersbourg afin de faire réaliser une étude sur les fonds nécessaires pour financer le chemin de fer ${ }^{82}$.

En 1856 la maison Rothschild reste un acteur incontournable dans les affaires ferroviaires russes, d'autant plus qu'elle constitue le troisième groupe concurrent des Pereire. Leurs liens en Russie et en Europe font d'eux des rivaux sérieux, au même titre que le groupe d'Oppenheim. Napoléon III et le comte de Morny soutiennent cependant les Pereire dans cette affaire, d'autant plus que l'empereur contribue à la fondation du Crédit mobilier pour créer un contrepoids à James Rothschild. La rivalité Pereire - Rothschild dans les chemins de fer russes est un nouveau chapitre d'une concurrence profondément ancrée dans les affaires industrielles françaises et c'est sous cet angle que nous devons l'observer.

54 Le contexte de fin de guerre donne une dimension géopolitique à la rivalité entre Rothschild et Pereire. Le Congrès de Paris fait des chemins de fer russes un sujet diplomatique entre la Russie et les puissances européennes, tout comme Gorčakov instrumentalise l'affaire pour favoriser sa politique de rapprochement avec la France. 
V. Boutenko signalait comment « en faisant donner la concession aux Pereire, Gortchakof voulait porter un coup à l'Angleterre dans la personne des Rothschild. Ceux-ci se vengeaient en brisant le plan diplomatique auquel il avait travaillé pendant plusieurs mois $»^{83}$. Le comte de Morny, qui veut soutenir des investisseurs proches de Napoléon III et créer des conditions favorables pour un rapprochement franco-russe, est du même avis. Ainsi, les intérêts privés de l'ambassadeur français font de lui un rival d'autant plus redoutable pour les Rothschild qu'il est lui même actionnaire du Crédit mobilier.

Le déplacement de la concurrence ferroviaire en Russie se traduit, comme nous l'avons vu pour le cas des Oppenheim, par une correspondance active montrant l'enchevêtrement des enjeux économiques et politiques. Entre les investisseurs et le GUPS, une bataille d'arguments techniques et financiers s'engage pour déterminer qui peut remporter la victoire. Ainsi, Joseph Paxton ${ }^{84}$ envoie, en accord avec les Rothschild, un argumentaire sur leurs propres forces et sur les vices de leurs rivaux, similaire à celui dressé par les Oppenheim.

Le 6 juillet 1856, deux mois avant que les Pereire et Oppenheim n'arrivent personnellement en Russie, les Rothschild annoncent l'arrivée à Saint-Pétersbourg de leurs ingénieurs. Leur objectif n'est pas seulement d'établir un réseau d'influences pour s'imposer mais, surtout, de mener des études de terrain afin de déterminer la viabilité des chemins de fer russes ${ }^{85}$. Contrairement à ce que nous observerons dans le cas des Pereire et de leur allié Stieglitz, les Rothschild n'établissent pas de lien avec Čevkin. Ils décident de négocier directement avec les ministres d'Alexandre II, comme le montre leur correspondance. Le 6 juillet 1856, J. Paxton, sous l'impulsion d'Anthony Rothschild, entre en contact avec le chancelier d'État $K$. Nesselrode, ancien ministre des Affaires étrangères ${ }^{86}$, et l'informe « qu'Edwin Clark est un ingénieur anglais de première catégorie et qu'il est lié à M. Robert Stephenson qui, comme Votre Excellence le sait, est lui aussi un grand ingénieur de ce pays ${ }^{87}$.

La maison Rothschild se veut une puissante référence ferroviaire au niveau mondial, et non pas seulement un symbole de stabilité financière, comme les Oppenheim. Le but des Rothschild est de convaincre la partie russe de la solidité de leur positionnement global par rapport aux Pereire. Dans cette mesure, ils affirment avoir «construit quelques milliers de miles de chemins de fer en Angleterre, plusieurs milliers en France, sur le Continent et en Amérique $»^{88}$. Ils promettent alors de réunir la somme qui serait nécessaire pour la construction des chemins de fer russes. En même temps, ils sont persuadés de pouvoir arriver à les construire entre 20 et $30 \%$ moins cher qu'en Europe.

Les arguments de J. Paxton dépassent largement le domaine des chemins de fer et se situent à mi-chemin entre le personnel et le professionnel, afin de convaincre K. Nesselrode de donner son appui aux investissements anglais. Dans son espoir de gagner un allié indispensable, il développe un argumentaire fleuri qui passe étonnamment de l'industrie ferroviaire à l'horticulture ${ }^{89}$. Il annonce qu'il se dispose à aller au Crystal Palace pour contempler les fleurs exotiques des environs et lui promet de ramener avec lui des experts horticulteurs en Russie ${ }^{90}$. Cette mention est un argument bien réfléchi de la part de Paxton puisque "parmi ses autres vocations [...] [Nesselrode] avait un grand intérêt pour l'horticulture $»^{91}$. Les Rothschild cherchent à se rapprocher de ce ministre en même temps que les Pereire d'A. Gorčakov.

Mais comme Gorčakov est devenu le nouveau ministre des Affaires étrangères, James Rothschild est conscient que les lettres de Paxton ne sont pas suffisantes s'il veut s'imposer. Il prend l'initiative d'écrire directement au président du Comité des ministres, 
le comte Orlov, et lui demande à l'occasion de se souvenir que les Rothschild avaient déjà formulé des propositions au gouvernement russe, bien avant les frères Pereire. Et il précise :

Je prends la liberté, d'après le désir que vous m'avez témoigné, de vous envoyer la note que je remis, le 15 novembre 1852 à S.M. l'Empereur Napoléon sur l'établissement de la Société de crédit mobilier [...] je n'ai aujourd'hui rien à changer à son contenu. ${ }^{92}$

Passant à l'offensive, il dénonce le danger que représentent les Pereire pour la finance mondiale, dans la mesure où « toutes les fois que les Gouvernements nous ont consultés, nous avons signalé avec la plus grande force, les dangers de ces institutions de crédit ${ }^{93}$. Nous observons ici comment les rivalités internes au monde de la finance française sont transposées au cas russe. Non content de prendre en personne la direction du groupe d'influence anglais en Russie, James Rothschild répète ses critiques dans deux autres lettres ${ }^{94}$.

61 De leur côté, les Pereire entrent volontiers dans la compétition avec les Rothschild. L'accueil que le Crédit mobilier rencontre en Russie les assure de remporter la victoire. Adolphe Eichthal, vice-président du Crédit mobilier, écrit alors à Stieglitz pour qu'il fasse plier Čevkin, jugé trop à l'écoute des Rothschild. Face au rôle actif qu'assume James Rothschild, Eichthal exprime sa préoccupation au sujet des résultats que cela pourrait avoir pour les intérêts de Pereire et associés :

quel changement depuis lors! Dès que nous avançons, comme toujours, Rothschild avance avec l'intention bien formelle de nous empêcher de conclure, avec celle beaucoup moins certaine de conclure lui-même, à moins de circonstances tout à fait exceptionnelles [...]. Toutes les fois qu'il [Émile Pereire] me soumet une proposition, quinze jours après Rothschild arrive avec le même projet. ${ }^{95}$

La concurrence ne se livre pas seulement au niveau de l'influence que les investisseurs français ont sur le gouvernement russe. Ainsi, dans le domaine de l'ingénierie, chacun des acteurs fait avancer ses pièces pour obtenir l'appui du Corps des ingénieurs des voies de communication russe. Les Rothschild placent leurs ingénieurs sous la protection de certains membres du gouvernement russe, comme nous l'observons dans une lettre qu'Anthony Rothschild fait parvenir depuis Londres à Aleksandr Gorčakov. Il annonce que « le Colonel Malcom ira à Saint-Pétersbourg accompagné de 4 ingénieurs, afin de remettre au Gouverneur général les propositions de certains grands capitalistes (les Rothschild inclus) qui désirent d'entreprendre la construction des chemins de fer en Russie $\aleph^{96}$. L'arrivée des ingénieurs anglais se fait autour d'avril 1856, quand Edwin Clark est déjà en route pour la Russie ${ }^{97}$. James Rothschild se sent fort du concours "d'ingénieurs éprouvés pour étudier sur les lieux les principales lignes que le gouvernement impérial veut entreprendre en les concédant à l'industrie privée ${ }^{98}$.

Dans ce système de concurrence à caractère géopolitique, les investisseurs avec lesquels les Pereire rivalisent en 1856, les Rothschild et les Oppenheim, vont devenir les principaux contradicteurs de la GSCFR. La jalousie qu'éveille le futur succès des Pereire est la source principale des critiques. Cependant, les hésitations de James Rothschild durant les négociations s'avèrent bien plus pertinentes que ce que nous pourrions imaginer. Il est probablement l'un des seuls à comprendre les dangers d'un tel investissement et la conjoncture défavorable dans laquelle cette affaire devait se réaliser. La dévaluation du rouble et l'effroi des bourses européennes porteraient un coup fatal au capital de ladite entreprise. Les spécialistes de la question ferroviaire russe n'ont vu dans les positions critiques des Rothschild qu'une affaire de fierté blessée et de politique 
internationale ${ }^{99}$. Cependant ils ont négligé la finesse du regard porté par James Rothschild sur l'image idyllique que ses contemporains se faisaient du potentiel économique russe.

Ceci est d'autant plus pertinent que l'avis de l'ingénieur Jullien, envoyé en Russie par $\mathrm{J}$. Rothschild, s'avère déterminant pour l'engagement de ce groupe. Nous apprenons par exemple qu'en juin 1856, c'est-à-dire au moment le plus critique des négociations, le baron Brunnov ${ }^{100}$ reçoit une dépêche secrète qui montre la prudence de J. Rothschild et de son ingénieur :

d'après l'avis du Comte Orlov, le Général Tchevkine [Čevkin] Vous prie de dire à Rothschild que M. Julien [Jullien] est trop indécis, que s'il ne veut pas perdre une occasion unique il faut qu'il envoie au plutôt [sic] des propositions définitives, car les concurrents en ont déjà présenté. ${ }^{101}$

James Rothschild comprend alors que le gouvernement russe veut favoriser la concurrence comme seul moyen de garder une marge de manœuvre face aux investisseurs. Devant cet aveu de faiblesse, le banquier se met momentanément en retrait et fait répondre au GUPS, le 3 juillet, qu'il est absent et qu'en son absence, ses fils ne sont pas en mesure d'élaborer des propositions définitives ${ }^{102}$.

Les hésitations dont fait preuve James Rothschild annoncent les principales causes de la faillite de la GSCFR. Bien qu'il soit tenté d'entreprendre la construction du réseau, lui et Jullien sont bien plus au courant de ce qui se passe en Russie, que les concurrents. D'une part, ils arrivent à la conclusion qu'un sujet si sensible pour l'Empire russe doit compter avec le concours des secteurs clés du pays. Sans eux, l'entreprise risquerait de s'affaiblir politiquement et cela la rendrait vulnérable à toutes sortes d'attaques. Rassurer les Russes est l'une des propositions les plus pertinentes du groupe Rothschild. En effet, en cas d'échec, les responsabilités politiques peuvent être partagées avec les fonctionnaires et les investisseurs russes. De cette manière

M. James Rothschild, M. Laing et M. Jullien, tous les trois s'accordent à l'unanimité pour dire que l'entreprise, pour réussir, doit porter essentiellement le caractère d'une combinaison russe. À ce point de vue, ils reconnaissent l'importance et nécessité indispensable du concours des capacités financières et intellectuelles de notre Pays [...]. Je remarque dans son esprit (Roth.) une certaine fluctuation, qui résulte naturellement de l'état monétaire du marché [...]. Je le vois disposé à tenter une entreprise à laquelle il met d'autant plus d'amour propre, qu'il y est intéressé par un sentiment de rivalité envers les chefs du Crédit mobilier. ${ }^{103}$

67 À part leur envie d'associer des Russes à leur entreprise, les Rothschild arrivent à la même conclusion que les Pereire: il faut donner une unité au réseau. Pour cela, ils proposent le rachat de la ligne Nikolaevskaja pour qu'elle devienne le chemin de fer central de la Russie puisque " l'unité de l'entreprise fera sa force et garantira son succès » ${ }^{104}$. Dans les négociations avec les Pereire, le gouvernement russe se méfie du rachat de cette ligne par les ennemis de la veille. Les Rothschild ont plus de chances de voir cette proposition acceptée dans la mesure où, de facto, la ligne Nikolaevskaja resterait aussi dans les mains d'investisseurs russes.

Le mois de septembre 1856 devient le moment culminant des négociations, quand le poids politique des concurrents semble paradoxalement le plus équilibré. En même temps que les Rothschild s'empressent de convaincre le gouvernement russe, le comte de Morny arrive en Russie en tant qu'ambassadeur de France. Le groupe Rothschild s'affaiblit au point que seuls les Oppenheim et les Pereire restent dans la course. L'ambassadeur de Prusse en Russie profite de la nouvelle situation pour informer Berlin que le 
gouvernement russe accorderait probablement l'une des lignes en projet au groupe germano-belge ${ }^{105}$.

La proximité du comte de Morny avec Gorčakov et la politique de rapprochement avec la France deviennent les éléments décisifs des négociations. En octobre 1856, Gorčakov présente à l'empereur un rapport dans lequel il penche pour les Pereire. Sans donner d'explications techniques, le choix des investisseurs français peut s'avérer éventuellement plus avantageux politiquement et économiquement dans la politique internationale russe. Devant le poids que représente alors le ministre des Affaires étrangères, et devant le Comité de ministres, Alexandre II choisit le groupe des Pereire quatre jours après la remise du rapport en question. Une lettre de l'ingénieur Jullien à Čevkin du 4 octobre 1856 indique que des rumeurs sont parvenues selon lesquelles le Gouvernement russe se serait déjà mis d'accord avec Stieglitz, Hope et Pereire pour la fondation de la GSCFR ${ }^{106}$. Cette victoire ouvre tout un chantier commercial entre la France et la Russie, car la politisation de la question ferroviaire déborde sur d'autres domaines. Des spécialistes français vont plus loin et insistent sur le rôle du comte de Morny dans la recherche de tarifs douaniers préférentiels, puisque

le grand-duc Constantin, qui gérait le ministère de la Marine russe, adressa toutes ses commandes à des maisons françaises. Morny s'empressa d'engager des négociations sur la baisse des droits de douane en Russie pour les produits français et le gouvernement de Saint-Pétersbourg fit à ces démarches un très bon accueil. ${ }^{107}$

La préférence pour des entrepreneurs français représente alors, non pas la fin de la concurrence pour la domination du territoire russe, mais une nouvelle étape dans la rivalité franco-anglaise. La victoire des Pereire éveille immédiatement des critiques en Angleterre et en France, auprès des journaux proches des Rothschild. Ces critiques auront des conséquences économiques sérieuses pour la GSCFR, d'autant plus que la concurrence entre les maisons financières pousse les investisseurs à entrer dans un système de spéculation. L'internationalisation des enjeux liés à la maîtrise du territoire impérial se poursuit. Elle s'exprime entre 1854 et 1856 par le biais des canaux diplomatiques mais, à partir de 1857 et jusqu'en 1862, elle prend une forme nouvelle: désormais, la presse prend le relais et les journaux deviennent des tribunes politiques en Russie et à l'étranger. Le terrain de la confrontation se déplace vers la scène publique après la fondation de la GSCFR. La presse française informe à partir de 1857 que,

depuis quelque temps, divers organes de la presse anglaise, et particulièrement le plus important des journaux de Londres, emploient leur talent et dépensent leur crédit avec une prodigalité vraiment extraordinaire à décrier le grand travail que vient d'entreprendre le gouvernement russe pour faire entrer son pays dans le système général de l'Europe en créant sur son territoire un vaste réseau de chemins de fer. ${ }^{108}$

\section{Les Pereire et alliés face à la concurrence}

Les Pereire essaient justement d'éviter d'entrer dans un système de compétition, ils craignent que cela puisse les pousser à faire des propositions imprudentes afin de s'imposer. En effet, ils utilisent toutes les armes politiques à leur disposition pour faire pression sur l'État russe afin de briser la concurrence. Dans un rapport du Comité d'investisseurs de la GSCFR (Baring, Borski, Pereire et Thurneyssen) du 2 août 1856, ils essaient de comprendre les intentions du gouvernement russe "et qu'on sache s'il a l'intention de traiter directement avec la société sans entrer dans un système de 
concurrence $»^{109}$. Les Pereire exercent des pressions sur l'équipe négociatrice que dirige Čevkin, sous prétexte que la concurrence ne fait que mettre en péril l'avenir de la GSCFR. Plusieurs dirigeants russes sont d'accord avec eux puisque «la spéculation pourrait s'exercer au détriment de la Société qui aurait la première couru les risques du nouveau système d'entreprise [les sociétés privées d'actionnaires $(\mathrm{NdA})] »^{110}$. Au moment où le politique devient central, l'idée d'un monopole dans l'industrie ferroviaire russe apparait comme un moyen de garantir les investissements.

72 À partir d'août nous observons une montée aux enchères de la part de tous les concurrents, ce qui pousse les Pereire à augmenter leur pression sur le gouvernement russe pour conclure rapidement l'affaire. Leur correspondance avec Stieglitz indique que, le $1^{\text {er }}$ septembre 1856, Thurneyssen, Pereire et Hottinguer partent pour la Russie afin d'avoir des entretiens directs avec différentes personnalités russes. Les négociateurs français insistent sur le caractère monopolisateur que devrait avoir leur compagnie dans le but de prévenir toute sorte de concurrence de la part de James Rothschild ou d'autres investisseurs européens. Ils poussent le gouvernement à accepter leurs conditions par leur capacité à apporter de véritables propositions dont les autres concurrents manquent :

Telles sont, Monsieur le Baron, les modifications qu'il nous a été possible d'apporter à nos propositions primitives. Ainsi modifiées, nous les croyons indispensables au succès d'une entreprise dont la grandeur est telle que nous ne l'aurions pas abordée si, par notre association, nous ne nous étions pas assuré le concours des principales places de l'Europe. ${ }^{111}$

Face à cette nouvelle situation, Čevkin comprend qu'il n'est plus le maître de la situation et que plusieurs intérêts sont en jeu entre certains ministres, le tsar et les investisseurs européens. Pour le compte du GUPS, Tegoborskij juge négativement la position de la GSCFR en des termes explicites. Dans une lettre indiquée comme "très confidentielle ", Tegoborskij écrit le 5 octobre 1856 que «c'est en partant de ce point de vue que j'entre dans les explications qui suivent tant en ce qui concerne le côté matériel que sous le rapport moral et politique de la question $»^{112}$. Fin connaisseur des forces productives de la Russie, il se livre à une analyse sur l'avenir de la question ferroviaire qui contredit l'assurance de certains milieux russes. Il argumente que Čevkin

exagère un peu la perspective brillante des chemins de fer en Russie, au point de vue des avantages financiers de l'entreprise comme des Compagnies qui s'en chargeraient. C'est l'un des principaux points sur lequel nos opinions diffèrent et je tiens à me justifier.

Rothschild et Tegoborskij prévoient que la GSCFR fera probablement faillite et qu'il est irresponsable de s'engager auprès du gouvernement russe comme le font les Pereire.

Tegoborskij continue dans sa lettre: «nos chemins de fer ne rapporteront longtemps en moyenne que $4 \%$ de revenu net $»^{113}$. Le GUPS observe donc avec préoccupation comment les pressions qu'exercent les Pereire risquent de pousser l'État à soutenir financièrement la future GSCFR pendant les années à venir. Le constat des risques que comportent les négociations et l'arrivée du groupe des Français en Russie deviennent une affaire d'État. Le GUPS se voit rapidement dépassé par la dimension politique de l'affaire et se retrouve dans un piège puisqu'il ne peut pas rejeter les propositions des Pereire. Tegoborskij se charge de faire comprendre à Čevkin sa place dans cette affaire. Il prévient le GUPS des conséquences d'un échec dans les négociations puisque,

lorsque des chefs de maisons considérables comme ces Messieurs, et dont l'un est à

la tête d'une des plus puissantes institutions de crédit en France, abandonnent leurs 
affaires pour venir traiter d'une grande entreprise avec le Gouvernement Russe et qu'après avoir fait le voyage et perdu quelques semaines d'un temps précieux (car pour eux time is money) ils échouent dans leurs négociations, ils partiront aigris au dernier point [...] ne les ayant pour nous, nous les aurons contre nous. ${ }^{114}$ d'enchérisseur dans un jeu qu'elle domine à peine. Devant la prudence dont fait preuve le gouvernement russe, les Pereire décident de passer en force avec leur voyage en Russie et de mettre fin aux propositions de la concurrence. Une lettre de Baring ${ }^{115}$ à Stieglitz du 6 août 1856 laisse entrevoir comment les investisseurs étrangers amènent le tsar à faire pression sur ses fonctionnaires. Pour eux, la priorité est d'arriver à un accord immédiat avec le GUPS sous menace de se retirer de la compétition: "vous nous permettrez peut-être de vous exprimer notre désappointement sur la position actuelle de la négociation avec le Gouvernement Imperial pour la concession de la construction et de l'exploitation des chemins de fer en Russie $»^{116}$. Il fait planer la possibilité d'une mise en veille de la modernisation des transports russes après la guerre de Crimée.

Baring passe ensuite à l'attaque contre le GUPS. Il affirme, après réception d'une dépêche très hostile de la part de Čevkin, que

pour que vous puissiez apprécier entièrement la cause des regrets que nous a fait sentir le ton de la dépêche, nous rappellerons en peu de mots à votre mémoire les circonstances dans lesquelles nous prîmes part aux négociations pendantes. ${ }^{117}$

Ce n'est à l'époque un secret pour personne que la Russie a perdu la guerre à cause de la déficience de ses transports et que les Pereire ont commencé à négocier avec le gouvernement russe avant même la signature de la paix. Baring veut éviter de faire des offres dangereuses et, poursuit-il,

nous devons avouer franchement que nous ne voyons dans la lettre de Monsieur le Général Tchevkine [Čevkin] aucune expression distincte du désir de traiter avec nos collègues ou avec nous-mêmes, ni aucune évidence que les communications antérieures avaient un autre but que le désir d'obtenir des informations utiles [...]. Il faut que le Gouvernement commence par prouver, par la garantie qu'il donne, qu'il a lui-même confiance dans le résultat [de la GSCFR]. ${ }^{118}$

79 La lettre de Baring vise à faire pression, non pas sur son associé Stieglitz, mais sur le gouvernement russe puisque, écrit-il, « nous désirons, si vous ne vous y opposez pas, que le contenu de cette lettre fût communiqué au Gouvernement impérial » ${ }^{119}$.

L'empereur et son entourage dirigent étroitement l'appel d'offres, qui ne passe pas directement par le GUPS. Cependant, l'hostilité que commence à manifester le directeur du GUPS met en péril la conclusion d'un accord avec les Pereire, un projet que le gouvernement russe n'est pas en mesure de rejeter. Les Français sont très surpris de voir les entraves que fabrique Čevkin dans les négociations et ne prennent pas la mesure de l'hostilité qu'ils éveillent en Russie. Ils décident de faire une épreuve de force et menacent de quitter la Russie sans avoir signé d'accord, tout en informant le gouvernement de leurs difficultés. La réponse ne se fait pas attendre de la part du tsar, qui rappelle à l'ordre le GUPS et brise les entraves au processus de formation de la GSCFR. La position d'Alexandre II est cependant ambiguë. Dans ses décisions, il semble vouloir satisfaire tous les secteurs concernés par les chemins de fer, au point qu'il ordonne la construction d'un réseau par des entreprises étrangères mais, en même temps, il place à la tête du GUPS un homme hostile à cette mesure. À plusieurs reprises, les faits montrent une volonté de tempérer cette réforme dans le domaine ferroviaire. De la sorte, Gorčakov informe Čevkin : 
[Il] m'est resté la même impression que vous. Effrayés par la crise financière [...] ils regrettent même les conditions qu'ils nous ont faites. Cela me semble une double raison pour nous de ne pas les laisser partir sans chercher à conclure. En concluant, ils laissent en garantie, je pense, beaucoup d'argent. ${ }^{120}$

81 Les forces combinées de l'ambassade de France et des maisons financières font plier le GUPS et son directeur. Le tsar lui fait savoir qu'il ne peut laisser partir les Français sans un accord et Čevkin comprend que plusieurs ministres appuient cette décision ${ }^{121}$. Il cesse alors d'entraver l'avancement des négociations et répond à Stieglitz le 9 août 1856 qu'il apprend «avec autant d'étonnement que de regret que la communication que j'ai été à même de Vous adresser Monsieur [...] a été prise par Vous comme étant dans un sens essentiellement différent de celui dans lequel elle avait été écrite ${ }^{122}$, d'où son invitation aux Pereire à entrer en pourparlers directs à Saint-Pétersbourg. Il opère un changement de ton pour exprimer qu'il est " pénétré du profond désir de conduire à une prompte et bonne fin l'affaire $»^{123}$.

Les Pereire réussissent à mettre en place de véritables mécanismes d'influence en Russie dans lesquels s'entremêlent des intérêts politiques corporatifs (les ingénieurs), nationaux (des ministres et une partie de l'opinion publique russe et française), régionaux (une alliance de maisons financières) et globaux (l'intérêt de l'État français). L'État russe n'a pas d'autre issue que d'abandonner le rôle d'arbitre qu'il veut jouer puisque, cerné par les intérêts étrangers, il doit devenir un acteur.

\section{Le gouvernement russe, acteur politique et modérateur dans les négociations}

Les chemins de fer russes ne sont pas seulement l'objet d'une rivalité internationale ni l'apanage des puissances internationales. Cela explique que le gouvernement ouvre la possibilité de négocier une partie de sa souveraineté au nom d'un transfert de technologies. Il se place en arbitre de la compétition mais, en même temps il négocie une partie de ses prérogatives. L'objectif est alors la mise en relation des provinces impériales entre elles par les moyens de communication les plus modernes de l'époque.

Dans cette stratégie à laquelle se livrent Alexandre II et Gorčakov après la guerre de Crimée, l'engrenage des négociations se construit autour de Čevkin, fervent défenseur des intérêts russes. Avec lui, le tsar prend bien garde de laisser à ce poste clé l'un des opposants à l'intervention des capitaux étrangers dans les voies de communication russes. Il cherche probablement à donner aussi des garanties aux opposants russes à la création de la GSCFR. L'opinion que se font de Čevkin A. Gorčakov et P. Valuev ${ }^{124}$, par la suite ses opposants au sein du gouvernement, montre bien le tempérament du directeur du GUPS. Petr Valuev est explicite dans son journal quand il fait référence aux positions de Čevkin au sujet des chemins de fer. Il le décrit ainsi, dans une réunion du Comité des finances du 14 mai 1868 : "Čevkin, l'infaillible mauvaise fée de tous nos contes officiels [en français $(\mathrm{NdA})$ ], continue à se serrer aux côtés des producteurs traditionnels d'alcool ${ }^{125}$. Ensuite, il transcrit un poème ${ }^{126}$ critiquant les entrepreneurs proches des slavophiles V. Kokorev ${ }^{127}$ et A. Košelev ${ }^{128}$. Les rivalités en question se répercutent et se prolongent au sein du gouvernement russe et perdurent au-delà de la période.

La perspective d'une faillite de la GSCFR laisse envisager un éventuel rachat avantageux pour les caisses de l'État. L'avenir de la GSCFR est loin d'être assuré et le gouvernement 
veut à tout prix impliquer les Pereire, Oppenheim et Rothschild, avant qu'ils ne se rendent compte des risques qu'une telle entreprise représente dans la conjoncture défavorable de la période. La dévaluation rampante du rouble, due à l'effort de guerre, rend instable l'environnement financier en Russie et rend la formation du capital de la GSCFR volatile ${ }^{129}$.

La correspondance entre Tegoborskij et Čevkin nous donne une idée des véritables priorités du GUPS. Elles permettent d'observer en quoi l'État russe est finalement moins arbitre qu'acteur dans cette affaire. Dans un rapport du 5 octobre 1856, marqué comme «très confidentiel », Tegoborskij fait part à Čevkin des étapes à suivre dans les négociations. Il livre alors des informations précieuses quant au contexte des négociations durant les mois de juin à octobre. Le GUPS constate que, en dépit de l'appel d'offres, l'arrivée des investisseurs en Russie non seulement crée des pressions politiques, mais elle risque aussi de mettre en péril le projet ferroviaire lui-même :

les idées de deux de ces Messieurs en ce qui concerne les avantages aléatoires de cette entreprise se sont beaucoup modifiées depuis qu'ils sont ici et qu'ils ont vu les choses de plus près [...]. Ils sont tous rebutés par les difficultés qu'ils ont rencontrées et auxquelles ils ne s'attendaient pas. ${ }^{130}$

87 L'État russe cherche à trouver un équilibre entre la fermeté et la réalité, dans la mesure où il veut montrer qu'il contrôle les négociations. Cependant, ses fonctionnaires sont bien conscients qu'un échec serait grave pour le développement de l'empire et pourrait se traduire par de nouvelles défaites militaires à l'avenir. Le GUPS décide alors de mieux se servir des rivalités entre les puissances mondiales. En effet, le prestige que donnerait la fondation de la GSCFR au vainqueur devient une arme pour l'État russe. Les investisseurs

n'y tiennent encore que par un certain point d'honneur ou si Vous voulez, par des considérations d'amour propre, car après s'être engagés dans cette affaire au point d'être venus ici, il leur répugne d'aboutir à un fiasco. Profitons donc de ces dispositions sans tendre la corde au point qu'elle rompe et elle est déjà, croyez-moi, très tendue. ${ }^{131}$

88 Si la situation russe semble si difficile aux yeux des hauts fonctionnaires, le mot d'ordre est de profiter de la conjoncture pendant que les investisseurs sont toujours dans la course.

Le GUPS se trouve dans le dilemme de vouloir garantir l'exécution rapide des travaux, sans confisquer à la future compagnie les moyens économiques de le faire. Il veut créer un compromis entre la souveraineté de l'État et les réalités financières de la compagnie. Mais l'État est prudent face aux garanties que les investisseurs attendent de lui. Tegoborskij signale que Čevkin ôte les moyens d'exécution de l'entreprise et mentionne que la garantie d'État est, en partie, un moyen d'attirer l'attention des Européens, plus qu'un support économique en cas de besoin. Tegoborskij comprend bien que les difficultés économiques peuvent compromettre la solvabilité même de l'entreprise, d'où le désir de garantir avant tout la construction des lignes. Il écrit ainsi à Čevkin «qu’à force de prudence, de prévision et de précaution nous risquons de perdre cette précieuse garantie en la sacrifiant à une garantie stipulée par écrit qui ne serait qu'une lettre morte si les moyens d'exécution manquent à ceux qui s'en chargeraient $»^{132}$. Le temps n'est plus aux barrières dressées contre l'arrivée massive de capitaux étrangers, mais à la captation de ceux-ci avant qu'ils ne se tournent vers d'autres horizons.

L'internationalisation de la question ferroviaire russe est au cœur de la période de réformes d'Alexandre II. Elle apparaît dans les négociations avec les investisseurs 
étrangers et dans la manière dont le politique prend une place prépondérante autour et à l'intérieur de l'État. Le dilemme auquel la victoire des frères Pereire pousse le gouvernement l'amène à vouloir encadrer la GSCFR dans les années suivantes. Il crée pour cette nouvelle compagnie des statuts qui posent les bases et servent d'exemple pour la construction des chemins de fer en Russie au XIX siècle.

Cependant les mécanismes qu'il mettra en place pour tempérer l'influence de la GSCFR vont se révéler bien plus efficaces que prévu, quand la GSCFR se verra assiégée en Russie de tous les côtés par les alliés de Čevkin. Le dilemme de la souveraineté divisera le gouvernement russe. Celui-ci sera placé au milieu d'une intense compétition entre intérêts rivaux. D'autre part, l'internationalisation de la question ferroviaire sera loin de prendre fin avec la publication des statuts en janvier 1857 ; une nouvelle étape s'ouvrira dans laquelle l'opinion publique prendra le relais. Des entrepreneurs russes de filiation slavophile, ainsi que certains membres du gouvernement et du GUPS, se livreront à une bataille dans les journaux de l'époque pour jeter le discrédit sur la compagnie française. Leur objectif sera de contenir l'influence croissante des intérêts étrangers sur ce qu'ils considèrent comme leur propre terrain d'influence. La solide position des Pereire ne cessera alors de se dégrader jusqu'à la faillite de leur société et à la renégociation des statuts en 1861. La GSCFR continuera d'exister, sous une forme bien plus réduite, sous l'emprise de l'État, et se videra de son contenu français. La publication des nouveaux statuts marqueront la mise en veille du réseau ferroviaire russe dans la décennie à venir.

\section{NOTES}

1. Nous avons choisi d'harmoniser les dates des sources russes citées dans cet article en adoptant le calendrier grégorien.

2. S.N., « Uspehi Rossii [Les succès de la Russie] », Vestnik promyšlennosti, 1859, nº 8, p. 155.

3. "Grande société des chemins de fer russes", Documents officiels, P.: Imprimerie administrative de Paul Dupont, 1861.

4. Glavnoe Upravlenie Putej Soobščenija.

5. En Russie, au RGIA (Rossijskij Gosudarstvennyj Istoričeskij Arhiv - Archives historiques d'État de Russie), le fonds 258 ; en France, les Archives Nationales de France ( $F^{14} 8636$ - Chemins de fer russes) et les Archives du ministère des Affaires étrangères (Correspondance commerciale, Saint-Pétersbourg, t. 30, 1856) complètent cette recherche.

6. Alfred Rieber publia le seul article véritablement important sur la GSCFR : Alfred Rieber, «The Formation of La Grande Société de Chemins de Fer Russes », Jahrbücher für Geschichte Osteuropas, 21, 1973, p. 375-391. D'autres historiens sont aussi incontournables pour notre sujet: Richard Mowbray Haywood, Russia Enters the Railway Age, 1842-1855, New York: Columbia University Press, 1998 ; Frithjof Benjamin Schenk, «Die Neuvermessung des Russländischen Reiches im Eisenbahnzeitalter ", in Jörn Happel, Christophe von Werdt, Mira Jovanović, éds., Osteuropa kartiert : Mapping Eastern Europe, Zürich - Berlin : Lit, 2010, p. 13-35. Parmi les études d'histoire économique, voir Thomas C. Owen, Dilemmas of Russian Capitalism: Fedor Chizhov and Corporate Enterprise in The Railroad Age, Cambridge : Harvard University Press, 2005 ; Thomas 
C. Owen, The Corporation under Russian Law, 1800-1917, Cambridge: Cambridge University Press, 1991.

7. La taille imposante du fonds 258 de RGIA, "La Grande Société des Chemins de Fer Russes », l'importance de ses inventaires et leur caractère quasiment inconnu aux historiens, nous ont poussé à accorder la priorité à leur dépouillement. D'autres fonds plus connus existent cependant en France, au Royaume-Uni et en Allemagne.

8. Cependant, d'autres compagnies privées apparaissent avant 1857. La compagnie de Varsovie Vienne (1848) en est un exemple, quoiqu'elle reste marginale par rapport à la taille de la GSCFR.

9. Il est membre du Conseil d'Empire, futur administrateur de la GSCFR jusqu'en 1858 et une autorité dans le milieu industriel en raison de sa publication : Ludwik Tegoborski, Études sur les forces productives de la Russie, P. : J. Renouard, 1852-1855, 4 vols.

10. Franck Yonnet, « La banque saint-simonienne, les "travailleurs" et les "capitalistes" : le projet des Sociétés mutuelles de crédit de 1853 des frères Pereire », Revue française d'économie, 13 (2), 1998, p. 59-99.

11. M. Aycard, Histoire du Crédit mobilier, 1852-1867, Bruxelles : A. Lacroix, 1867.

12. Guy Fargette, Émile et Isaac Pereire : l'esprit d'entreprise au XIX siècle, P. : L'Harmattan, 2001, p. 22.

13. Le lecteur peut trouver une illustration de la diamétrale opposition entre Pereire et Rothschild et de l'apparition de l'industrie ferroviaire en France dans: Maurice-Édouard Berthon, Émile et Isaac Pereire : la passion d'entreprendre, P. : Publication universitaire, 2007 ; Georges Ribeill, La révolution ferroviaire: la formation des compagnies de chemins de fer en France, 1823-1870, P. : Belin, 1993, p. 143.

14. Ludwik Tegoborski, Essai sur le crédit mobilier, Bruxelles : Hayez, 1856.

15. Pierre Larousse, Grand dictionnaire universel $d u$ XIX ${ }^{\mathrm{e}}$ siècle, P. : Administration du grand Dictionnaire universel, t. 8, 1866-1877, p. 1012, citant : «Des institutions du crédit mobilier, par Louis Tegoborskij, nouvelle édition augmentée de la vie de l'auteur, d'une introduction et des notes (1857)».

16. L'influence de l'ingénierie française est cependant bien antérieure. Elle remonte aux années 1810-1830, quand entrent au service du tsar des ingénieurs prestigieux comme Maurice Destrem, Émile Clapeyron, Gabriel Lamé ou Pierre-Dominique Bazaine: voir Irina Gouzevitch, Dmitri Gouzevitch, «Les contacts franco-russes dans le domaine de l'enseignement supérieur technique et de l'art de l'ingénieur », Cahiers du monde russe et soviétique, 34 (3), 1993, p. 345-368.

17. Aycard, Histoire, p. 342.

18. Archives du ministère des Affaires étrangères (AMAE), correspondances commerciales, Saint-Pétersbourg, $\mathrm{n}^{\circ}$ 30, 1856-1857, folio 224.

19. Aleksandr Stieglitz est à l'époque le propriétaire de la première banque de Saint-Pétersbourg et l'un des hommes forts du monde ferroviaire russe. Il est présent depuis plusieurs années dans diverses entreprises de chemins de fer, notamment par son apport financier dans la construction de la ligne de Peterhoff à Saint-Pétersbourg (1853). Entre 1840 et 1850 il réussit un grand emprunt en Europe, à des taux très convenables, pour construire le chemin de fer entre Saint-Pétersbourg et Moscou. Par la suite, il deviendra le vice-président du conseil d'administration de la GSCFR entre 1857 et 1858 et, en 1860, Alexandre II le nomme président de la Banque d'État russe. Voir par exemple A.M. Solov'eva, Železnodorožnyj transport Rossii vo vtoroj polovine XIX v., M. : Nauka, 1975, p. 74.

20. RGIA, f. 258, op. 8, d. 301, 1. 1. Lettre de A. Stieglitz du 7 septembre 1856 (en français).

21. Après avoir soutenu les Pereire face aux Rothschild, ces journaux, parmi lesquels Žurnal dlja akcionerov, Russkaja Beseda ou Vestnik promyšlennosti, deviennent en 1859 les principaux critiques de la GSCFR.

22. "Glavnoe obščestvo rossijskih železnyh dorog », Žurnal dlja akcionerov, 10 juillet $1858, n^{\circ} 79$, p. 674. 
23. Le comte Charles de Morny (il devint duc de Morny en 1862), était le demi-frère de Napoléon III. Le comte de Morny fut l'ambassadeur de France à Saint-Pétersbourg de 1856 à 1857. Son importance pour notre sujet ne tient pas seulement à son rôle pendant les négociations de paix à Paris, mais aussi au fait qu'il est un membre du conseil d'administration du Crédit mobilier des Pereire et l'un de ses actionnaires. Voir Aycard, Histoire, p. 84.

24. Jean Autin, Les frères Pereire : le bonheur d'entreprendre, P. : Perrin, 1984, p. 135.

25. RGIA, f. 258, op. 8, d. 297, 1. 40. Lettre de Emile Pereire à A. Stieglitz, Moscou, 15 septembre 1856.

26. Compagnie de chemins de fer française qu'absorbe dans les années 1850 le chemin de fer de Saint-Étienne - Lyon, Andrézieux - Roanne et Saint-Étienne - Loire.

27. Aycard, Histoire, p. 69.

28. C'est le moment le plus intense des négociations entre l'État et les entrepreneurs étrangers.

29. RGIA, f. 258, op. 8, d. 298, 1.5 (en russe). Ce rapport fait partie d'une série de documents qui doivent être pris en considération dans l'histoire ferroviaire russe. Leur richesse réside dans la manière dont ils montrent comment, en 1856-1857, l'État est traversé par un esprit de réforme dans le but de se donner de nouveaux moyens de transport.

30. RGIA, f. 258, op. 8, d. 296, 1.12 (en français).

31. RGIA, f. 258, op. 8, d. 296, 1. 14 (en russe).

32. Ibidem.

33. RGIA, f. 258, op. 8, d. 296, 1. 49.

34. Voir Alexandr von Gerstner, O vygodah postroenija železnoj dorogi [Des avantages de construire le chemin de fer], SPb.: Académie impériale des sciences, 1836; Narkis Tarasenko-Otreškov, Ob ustroenii železnyh dorog v Rossii [De la contruction du chemin de fer en Russie], SPb. : N. Greča, 1835 ; Pavel Mel'nikov, O železnyh dorogah [Du chemin de fer], SPb. : Direction des voies de communication, 1835.

35. Aleksandr Puškin, Polnoe sobranie sočinenij [Æuvres complètes], t.10: Pis'ma [Lettres], p. 615, $\mathrm{n}^{\circ} 770$, lettre de Puškin à V.F. Odoevskij sur le réseau de chemins de fer russes, novembre-décembre 1836.

36. Ce premier pont entre la Russie et l'Europe commence avec l'arrivée à Saint-Pétersbourg d'Augustin de Betancourt, Adolphe Bazaine, Émile Clapeyron et Gabriel Lamé. Voir Gouzevitch, "Les contacts franco-russes... », p. 345-368.

37. Leo von Zeebach, gendre du chancelier russe Karl Nesselrode, en voyage à Paris en 1856.

38. Alors président du Comité de ministres, il est l'un des hommes le plus proches d'Alexandre II.

39. C'est-à-dire bien avant que les négociations ne commencent officiellement.

40. RGIA, f. 258, op. 8, d. 296, l. 1. Ce dossier contient des correspondances entre les personnes qui se sont chargées à l'époque d'ouvrir l'appel d'offres à l'Europe. Il est important dans la mesure où il montre comment l'État russe n'a d'autre issue que de céder une partie de son autorité, pour la première fois, à une compagnie privée et étrangère.

41. Ibidem.

42. Directeur général des voies de communication entre 1857 et 1862 et ennemi principal de la GSCFR. Ami d'Alexandre II, il participe durant les années 1850 à la promotion du réseau ferré russe. K. Čevkin défend les investisseurs russes en raison de sa profonde hostilité envers les capitaux étrangers. Il devient alors un proche des milieux slavophiles et, en 1856, se fait le porte-parole d'entrepreneurs russes puissants comme Vasilij Kokorev, Ivan Mamontov et Fedor Čižov. Voir « Konstantin Vladimirovič Čevkin », Russkaja Starina, 1877, t. 19, p. 1-22

et « Konstantin Vladimirovič Čevkin », Russkaja Starina, 1885, t. 45, p. 365-384.

43. RGIA, f. 258, op. 8, d. 296, 1. 6. Texte en français.

44. Il est d'ailleurs le principal promoteur du télégraphe transatlantique et le premier président de l'American Telegraph Confederation. Voir son ouvrage principal: Tal. P. Shaffner, The 
Telegraph Manual : A Complete History and Description of the Semaphoric, Electric and Magnetic Telegraphs Of Europe, Asia, Africa, And America, New York : Pudney \& Russell Publishers, 1859.

45. Alfred Rieber mentionne la présence de cet entrepreneur dans Rieber, "The Formation... ", p. 380 .

46. Evgenij Lamanskij, Otkuda vzjat' kapitaly dlja postroiki železnyh dorog v Rossii ? [Où prendre les capitaux pour construire des voies de chemin de fer en Russie], SPb. : Voennaja Tipografija, 1865, p. 4.

47. Aycard, Histoire, p. 370.

48. RGIA, f. 1272, op. 1, d. 5, 1. 35. Lettre de T. Shaffner au comte Orlov du 2 août 1855 (en anglais). Ce dossier contient les négociations entre les Américains et le gouvernement russe pour la construction du réseau ferroviaire. Il faut noter le profond mécontentement avec lequel Shaffner critique l'indécision du gouvernement russe et la discrimination dont il fait l'objet par rapport aux investisseurs européens.

49. Ibidem.

50. Ibidem.

51. RGIA, f. 256, op. 1, d. 4, l. 1.

52. Pavel Petrovič Mel'nikov est l'un des constructeurs de la ligne Nikolaevskaja. Ministre des transports de 1865 à 1869, il obtint avec V. Kokorev la concession de la ligne Don - Volga, commencée en 1863. Voir G.M. Fadeev, Mel'nikov - inžener, učenyj, gosudarstvennyj dejatel' [Mel'nikov, ingénieur, savant et homme d'État], SPb. : Gumanistika, 2003.

53. RGIA, f. 1272, op. 1, d. 5, 1. 1. C'est-à-dire 9 millions de roubles de moins que ce que les Pereire offrent l'année suivante, mais avec une différence de taille : la concession ne devrait durer que 40 ans, tandis que celle des Pereire s'accorde pour 85 ans.

54. RGIA, f. 1272, op. 1, d. 5, 1. 49. Lettre de T. Shaffner au directeur du GUPS (en anglais).

55. Bien que la construction de la ligne Moscou - Saint-Pétersbourg montre un manque relatif d'ingénieurs dans les explorations puis dans la construction, le Corps d'ingénieurs des voies de communication est en mesure de fournir des hommes capables de mener à bien un tel projet. D'ailleurs, plusieurs ingénieurs reçoivent une expérience à l'étranger: P. Mel'nikov part aux États-Unis en 1839, M.S. Volkov part en mission en France (1835), K. Čevkin en 1836 et S.V. Kerbedz de 1838 à 1839. N.O. Kraft est envoyé en Autriche la même année. Voir à ce sujet Haywood, Russia Enters...

56. RGIA, f. 1272, op. 1, d. 5, 1. 116. Lettre de P. Kleinmichel à T. Shaffner du 9 septembre 1855 (en russe).

57. Ibidem, p. 59. Lettre du Comité pour les chemins de fer à T. Shaffner du 23 août 1855.

58. Industriel et homme politique belge, il est membre de la chambre des représentants de Belgique, jusqu'en 1857, et du Comité permanent du chemin de fer.

59. Rondo E. Cameron, France and the Economic Development of Europe, 1800-1914 : Conquests of Peace and Seeds of War, Princeton : Princeton University Press, 1961, p. 381.

60. RGIA, f. 258, op. 8, d. 301, 1. 14.

61. C'est à dire une ligne Moscou - Odessa et non pas Moscou - Théodosie.

62. Cette usine, appartenant à la Duchesse de Leuchtenberg (la sœur d'Alexandre II), est déjà en vente en 1855, puisque P. Kleinmichel en discute avec T. Shaffner durant le mois de septembre : voir RGIA, f. 1272, op. 1, d. 5, 1. 118.

63. RGIA, f. 258, op. 8, d. 301, 1. 11 (en français). Ce dossier contient les négociations entre l'État russe et les investisseurs belges et allemands. Il est d'autant plus important qu'il contient une partie des réponses qui amènent le gouvernement russe à choisir définitivement les Pereire.

64. Comme nous le verrons, ceci est commun aux autres investisseurs européens présents dans les affaires ferroviaires russes (Pereire et Rothschild).

65. RGIA, f. 258, op. 8, d. 300, 1. 1 . 
66. Son nom n'apparaît pas dans les archives russes (RGIA, f. 258) mais ses travaux sont conservés à la Bibliothèque nationale de France (BNF) : Adolphe Le Hardy De Beaulieu, Les Chemins de fer en Russie, état actuel des voies de communication et de transport dans la Russie d'Europe, Bruxelles : impr. de E. Guyot, 1857.

67. RGIA, f. 258, op. 8, d. 301, 1.9 (en français).

68. Ibidem, 1.3.

69. Ibidem, 1. 5. Lettre de Wendelstadt à K. Čevkin du 12 septembre 1856 (en français).

70. RGIA, f. 258, op. 8, d. 301, 1. 7. Télégramme de S. Oppenheim à K. Čevkin (en français).

71. Ibidem, 1. 18.

72. Ibidem, 1. 20. Lettre de Brower de Hogendorf à K. Čevkin du 10 octobre 1856 (en français).

73. Ibidem.

74. RGIA, f. 258, op. 8, d. 300, 1. 1. Lettre de Brower de Hogendorf à K. Čevkin du 9 juin 1856 (en français).

75. RGIA, f. 258, op. 8, d. 301, 1. 20. Lettre de Brower de Hogendorf à K. Čevkin du 10 octobre 1856 (en français).

76. Ibidem.

77. Ibidem. Lettre de K. Čevkin à Brower de Hogendorf du 16 octobre 1856 (en français).

78. Ibidem.

79. E.F. Kankrin, ministre des Finances de 1823 à 1844, fervent opposant à la construction ferroviaire, en raison de sa charge financière pour l'État russe.

80. L'État commence sa construction sous le règne de Nicolas $I^{\mathrm{er}}$ mais finit par la céder à la GSCFR en 1857.

81. Les Hope sont l'un des principaux actionnaires de la GSCFR en 1857.

82. Haywood, Russia Enters..., p. 540.

83. Victor Boutenko, «Un projet d'alliance franco-russe en 1856 », Revue historique, CLV (2), 1927, p. 319.

84. Un des ingénieurs de confiance des Rothschild et constructeur du Crystal Palace à Londres pour l'exposition universelle de 1851.

85. Le siège de la maison Rothschild à Paris, sous la direction de James Rothschild, est le plus réticent sur la rentabilité du projet russe, mais il est finalement convaincu de tenter sa chance quand il observe le dynamisme des Pereire en Russie.

86. Fonction qu'il occupa jusqu'à son remplacement, le 15 avril 1856, par A. Gorčakov.

87. RGIA, f. 258, op. 8, d. 299, 1.14 (en anglais). Comme les dossiers que nous avons jusqu'ici analysés, celui-ci fait partie de la correspondance du GUPS en relation avec la GSCFR.

Le delo 299 concerne les négociations entre le gouvernement russe et les Rothschild.

88. Ibidem.

89. Joseph Paxton est connu à l'époque pour être un fin jardinier et il est à la tête de nombreuses serres au Royaume-Uni.

90. Il n'est pas tout à fait étrange que leur correspondance tourne autour des chemins de fer et de l'horticulture, puisque l'exposition universelle de 1855 à Paris avait pour thème l'agriculture (dont un hectare dédié à l'horticulture), l'industrie et les beaux-arts : voir Michel Alcan, Visite à l'exposition universelle de Paris en 1855, P.: Hachette, 1855; Henri Moulin, Impressions de voyage d'un étranger à Paris : visite à l'Exposition universelle de 1855, P. : impr. de A. Lebel, 1856.

91. Harold Ingle, Nesselrode and the Russian Rapprochement with Britain, 1836-1844, Berkeley : University of California Press, 1976, p. 18.

92. RGIA, f. 258, op. 8, d. 299, 1. 31 (en français).

93. Ibidem.

94. Ibidem, 1.38-43: il s'agit de deux notes dans lesquelles sont répétées ses mises en garde contre des institutions de crédit par actions telles que le Crédit mobilier. 
95. RGIA, f. 258, op. 8, d. 296, 1.157. Lettre d'Adolphe Eichthal du 6 août 1856 à A. Stieglitz (en français).

96. RGIA, f. 258, op. 8, d. 299, 1. 3. Lettre d'Anthony Rothschild à A. Gorčakov du 5 mai 1856 (en français).

97. Ibidem, 1. 20.

98. Ibidem, 1. 6. Lettre d'Anthony Rothschild à A. Gorčakov du 23 mai 1856 (en français).

99. C'est le cas de Rieber, « The Formation... ».

100. Filip Brunnov, diplomate russe qui participe au Congrès de Paris en 1856.

101. RGIA, f. 258, op. 8, d. 299, 1. 57. Note anonyme du 30 juin 1856 (en français).

102. Ibidem, Télégramme du 3 juillet 1856.

103. Ibidem, 1. 60. Dépêche du baron Brunnov à D. Tolstoj le 12 août 1856 (en français).

104. Ibidem.

105. Boutenko, « Un projet... », p. 302.

106. RGIA, f. 258, op. 8, d. 299, 1. 77.

107. Boutenko, «Un projet... », p. 302.

108. Journal des Débats, 6 mai 1857, p. 1.

109. RGIA, f. 258, op. 8, d. 296, 1.137. Rapport du Comité des fondateurs de la GSCFR du 2 août 1856. Plusieurs indices dans ce dossier laissent comprendre à quel point le gouvernement russe perd l'initiative durant les négociations avec les investisseurs européens.

110. Ibidem.

111. Ibidem, l. 172.

112. RGIA, f. 258, op. 8, d. 297, 1. 128. Ces correspondances sont en russe. Le dossier contient des discussions internes au GUPS. Ils permettent de faire une analyse de la position politique de l'État russe face à d'autres puissances étrangères dans ce domaine.

113. Ibidem.

114. Ibidem.

115. Banquier allié aux frères Pereire.

116. RGIA, f. 258, op. 8, d. 296, 1. 145.

117. Ibidem.

118. Ibidem.

119. Ibidem.

120. RGIA, f. 258, op. 8, d. 297, 1. 139 (en russe).

121. Probablement A. Gorčakov, P. Valuev et P. Brok (ministre des Finances), comme le montre postérieurement P.A. Valuev, Dnevnik, t. 2 : 1865-1876, M. : Akademija Nauk SSSR, 1961, p. 264, 15 avril 1868.

122. RGIA, f. 258, op. 8, d. 296, 1. 158.

123. RGIA, f. 258, op. 8, d. 297, 1. 190.

124. Favorables à l'investissement massif de capitaux étrangers dans le domaine ferroviaire et à la vente du chemin de fer Nikolaevskaja à la GSCFR.

125. P.A. Valuev, Dnevnik, t. 2 : 1865-1876, M. Akademija Nauk SSSR, 1961, p. 263, 14 mai 1868.

126. On peut trouver ce poème dans A. Ostrovskij, Epigramma i satira : iz istorii literaturnoi bor 'by XIX veka [Epigramme et satire : histoire de la bataille littéraire au XIX ${ }^{\mathrm{e}}$ siècle], t. II, 1932, p. 104-105.

127. Vasilij Kokorev (1817-1889), vieux-croyant, l'un des principaux investisseurs russes dans le domaine ferroviaire. Il se rapproche des milieux slavophiles, notamment de F. Čižov, pour limiter l'influence de la GSCFR. En août 1858, il obtient la concession de la ligne Don - Volga avec P. Mel 'nikov.

128. Aleksandr Košelev (1806-1883), slavophile et figure importante dans le débat sur les chemins de fer à partir de 1857. Son journal Russkaja Beseda sert de tribune aux critiques principaux de la GSCFR. 
129. Voir à ce sujet les mémoires d'Evgenij Lamanskij, notamment sa description de la situation financière russe de 1856 à 1857: Evgenij Lamanskij, Vospaminanija 1840-1880 [Mémoires, 1840-1880], Penza : Redakcija žurnala « Zemstvo », 1995, p. 39.

130. RGIA, f. 258 , op. 8 , d. 297, 1.128 (en russe). Il s'agit d'un long rapport sur les questions politiques, économiques et techniques dans les négociations. C'est un document central pour comprendre le déroulement des faits durant la période.

131. Ibidem

132. Ibidem.

\section{RÉSUMÉS}

Cet article analyse le dilemme entre la modernisation des transports et la souveraineté de l'État dans la Russie du XIX ${ }^{\mathrm{e}}$ siècle. L'auteur identifie les raisons qui poussent le gouvernement russe, après la guerre de Crimée, à se tourner vers les ennemis de la veille pour la construction d'un vaste réseau ferroviaire. La perspective de fonder une compagnie de chemins de fer privée et étrangère, appelée "La Grande Société de Chemins de fer Russes", provoque un débat considérable dans la Russie d'Alexandre II, traversée par un besoin de réformes. L'article reconstitue l'opposition entre plusieurs organes de l'État et des groupes financiers, russes et étrangers, qui défendent la souveraineté de l'état ou l'arrivée de capitaux étrangers. L'utilisation de sources de cette compagnie, méconnues par les historiens, contribue grandement à la compréhension des mécanismes qu'utilisent les maisons financières européennes (Rothschild, Pereire et Oppenheim) pour influencer la politique d'Alexandre II dans ce domaine. Elles permettent de mieux comprendre l'internationalisation du territoire russe et les jeux de pouvoir qui influencent la modernisation des transports russes.

In this article, the author analyses the dilemma between transport modernisation and State sovereignty in nineteenth-century Russia. It identifies the reasons that drove the Russian state to appeal to its recent enemies of the Crimean war for the construction of a wide railway network. The idea of founding a private and foreign company, called The Main Society of Russian Railways, aroused an important debate at the beginning of Alexander II's reign, when the need for reforms became patent. The article identifies the antagonism between several official organs and financial groups, both Russian and foreign, which defended either the State's sovereignty or the arrival of European capitals. The use of the company's archives, almost unknown by historians, highly contributes to a better comprehension of the mechanisms used by European financial groups (Rothschild, Pereire and Oppenheim) to influence Alexander II's railway policies. They permit us to understand the internationalisation of the Russian territory and the games of power that influenced the modernisation of Russian railways.

\section{AUTEUR}

\section{JUAN CAMILO VERGARA}

Centre d'études des mondes russe, caucasien et centre-européen CNRS-EHESS, Paris. juancver@me.com 